\title{
Identification of Passive Knee Joint and Shank Dynamics in Paraplegics Using Quadriceps Stimulation
}

Henry M. Franken, Peter H. Veltink, Member, IEEE, Roel Tijsmans, Henk

Nijmeijer, Senior Member, IEEE, and Herman B. K. Boom, Associate Member, IEEE

\begin{abstract}
The dynamics of the knee joint-freely swinging lower leg system of three sitting paraplegic patients was identified using a parameterized analytical model. The system was excited by a knee torque, generated by randomized interpulse interval stimulation of the quadriceps. Surface electrodes were used for stimulation. The output of the knee joint-lower leg system was the angular position, velocity and acceleration. A measurement set-up using accelerometers was designed and characterized successfully. A model structure was taken from the literature and its complexity was subsequently minimized based on the experiments and off-line identification. The resulting model structure facilitates possible recursive identification. Model parameters of the lower leg dynamics were successfully estimated using a least-squares algorithm in combination with the LevenburgMarquardt algorithm, minimizing the error in estimated angular acceleration. The inter-subject variability in parameter values for the knee joint damping and the gravitational component appeared to be small when normalized to the inertia of the lower leg. A term, representing the nonlinear elastic properties in the knee joint, appeared to improve the prediction capability of the model significantly. The identified model for the knee joint and shank dynamics accurately predicted passive lower leg movements. The magnitude of active knee torque due to elicited quadriceps contraction could be estimated using the identified model.
\end{abstract}

\section{INTRODUCTION}

C ONTROL of Functional Electrical Stimulation (FES) for restoring functional lower-extremity movements in paraplegics is a very difficult problem, due to its nonlinear, timevarying, and unstable characteristics. Control performance could be enhanced if an adequate model of this musclelimb system would be available [1], [2]. Such a model could predict the state of the human muscle-limb system (angular position, velocity and acceleration) in FES-induced dynamic contractions on-line. It would, thus, enable the use of adaptive control principles to compensate for nonlinear and timevarying characteristics and to detect disturbances. To gain insight in model-based control strategies for the control of FES-induced paraplegic gait, knee-joint position control using quadriceps stimulation is currently being investigated [3].

Manuscript revised October 14, 1993. This work was partially supported by the European Fund for Regional Development (Enschede, The Netherlands).

H. M. Franken, P. H. Veltink, R. Tijsmans, and H. B. K. Boom are with the Department of Electrical Engineering, University of Twente, The Netherlands.

H. Nijmeijer is with the Department of Applied Mathematics, University of Twente, The Netherlands.

IEEE Log Number 9214850
In general, one can gain more insight in the system (to be controlled) and save a lot of time by computer modeling and simulation. Specifically, the performance of control strategies can be studied using computer simulation without involving the actual system. This is particularly interesting in the rehabilitation engineering, since experiments are sometimes burdensome for the participating subjects and are generally time-consuming.

Several authors have reported approaches on model-based control of stimulated paralyzed human extremities [1], [3]-[8]. One of the problems studied was the control of the knee joint by electrical stimulation of hamstrings and quadriceps [1], [7], [8]. Hausdorff and Durfee [1] tested an open-loop feed forward scheme to control the movement or the isometric torque of this system. They concluded that the control performance improved when off-line estimated parameterized nonlinearities in the muscles and the knee joint-lower leg system were accounted for, but did not report validation of their models and parameters. Hatwell et al. [7] developed a model reference adaptive controller to control knee joint position. The model consisted of a nonlinear part followed by a linear deterministic auto regressive moving average model. Controller performance improved when the model reference parameters were chosen, such that the closed-loop bandwidth was severely limited. However, frequent clamping of the control signal impeded validation of the reported models from the displayed registrations. Still, an identified model for the passive knee joint and shank dynamics, suitable for state prediction, and an appropriate identification methodology have not been reported as yet.

The dynamics of human joints is defined by relations between the angles between the connected limbs to the forces acting on it. These forces are caused by passive characteristics of the joints and limbs as well as the active contraction of associated muscles. The passive characteristics consist of mass and inertial properties of the limb, and damping, elasticity and stiffness components in the joint [2]. The contributions to joint torque due to these passive characteristics can be described using an inverse dynamic Newton-Euler model formulation, which is frequently used in modeling of robots [9]. This model can be identified using measurements or estimates of angular position, velocity and acceleration during passive movements.

Kearney and Hunter [2] reviewed several experimental studies on the identification of single human joint dynamics in alert human beings. Several model types, among which the inverse 


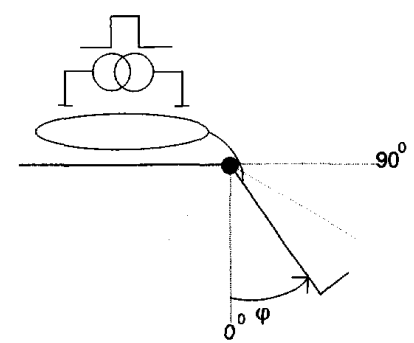

Fig. 1. Schematic drawing of the quadriceps-freely swinging lower leg system with surface stimulation of the quadriceps muscles. Knee angle $\varphi$ is defined in this figure, with $\varphi=0 \mathrm{deg}$, when the leg was at rest. Knee torque $M_{a}$ has the identical orientation. The knee angle range (dashed) in which the model should describe the system's behavior is approximately from -40 to $60^{\circ}$.

dynamic Newton-Euler model formulation as used in the current paper, were used to describe the joint dynamics. Excitation inputs were generally applied by external apparatus perturbing either the joint position or joint torque. Identification methods and results for the passive knee joint and shank dynamics in a freely swinging condition, as studied in this paper, were not reported. In recent work of Flaherty et al. [10], [11] and Robinson et al. [12], instrumentation, measurement and identification techniques for the identification of (nonlinear) models of the ankle joint during electrical stimulation of the soleus were reported. The ankle joint position and velocity were perturbed in .steps while measuring the torque at the ankle joint. In the approach, reported in the current paper, accelerometers were used to estimate joint torque due to electrical stimulation. This facilitated measurements during a freely swinging movement.

The objective of the current paper is the identification of passive knee joint and shank dynamics in paraplegics. Measurements for the identification were performed during freely moving conditions of the lower leg, induced by stimulation of the quadriceps muscles. A model structure was taken from literature. The complexity of this model was minimized and its parameters were estimated based on off-line identification. The resulting model showed good data fitting and accurately predicted passive lower leg movements. Furthermore, the magnitude of active knee torque due to quadriceps contraction could be estimated using the identified model.

\section{THEORY}

\section{A) Model Structure}

The considered quadriceps-lower leg system is schematically depicted in Fig. 1 . The knee angle was taken $0 \mathrm{deg}$ when the leg was at rest. The knee angle operating range, in which the identified model of the knee joint-lower leg dynamics, should accurately describe the system behavior was approximately between -40 and $60 \mathrm{deg}$. This angle range was chosen in conformity with a previous study on the control of the considered system [3].

The model to be identified represents the passive properties of the knee joint and lower leg. The excitation input is
TABLE I

SPECIFIC DATA ABOUT THE THREE PARTICIPATING PARAPLEGIC SUBJeCTS. THE SUBIECTS, ALL COMPLETE T5-T6 LEVEL SPINAL CORD INJURED PATIENTS, HAD BEEN ENROLLED IN THE FES TRAINING PROGRAM OF THE ROESSINGH REHABILITATION CENTER FOR THE RESTORATION OF LOCOMOTOR FUNCTIONS FOR A MINIMUM OF 6 MONTHS (SEE ALSO [18]). OT EXHIBITED SIGNIFICANT ACTIVE SPASM IN HIS RIGHT LEG, WHICH WAS THEREFORE NOT INCLUDED IN THE IDENTIFICATION EXPERIMENT

\begin{tabular}{ccccc}
\hline subject & sex & $\begin{array}{c}\text { year of } \\
\text { birth }\end{array}$ & $\begin{array}{c}\text { year of } \\
\text { injury }\end{array}$ & FES training \\
\hline TN & male & 1974 & 1989 & 15 months \\
JM & female & 1961 & 1981 & $\begin{array}{c}4 \text { years and 2 } \\
\text { months }\end{array}$ \\
OT & male & 1950 & 1991 & 6 months \\
\hline
\end{tabular}

active knee torque $M_{a}$, generated by quadriceps stimulation. A parameterized differential equation for a single joint-limb system often reported in literature [2], [13-16] is

$$
\begin{aligned}
M_{a}= & I \cdot \ddot{\varphi}+D \cdot \dot{\varphi}+F(\varphi) \\
F(\varphi)= & G \cdot \sin (\varphi)+K \cdot \varphi-k 1 \cdot\left(e^{-k 2 \cdot \varphi}-1\right) \\
& +k 3 \cdot\left(e^{k 4 \cdot \varphi}-1\right)
\end{aligned}
$$

Its parameters follow from the underlying biomechanics: inertia $(I)$, a gravity component $(G)$, damping $(D)$ and passive elasticity ( $K$ and parameters $k 1 . . k 4$ of the exponential terms). Thus, $M_{a}$ is the active torque at the joint. The exponential terms for the elasticity represent limiting nonlinearities in passive torque near the end stops. The parameters of (1) were assumed independent of $M_{a}$.

Kearney and Hunter [2] reported that models without an explicit nonlinear term for elasticity generally provide excellent description of human joint dynamics. Therefore, we examined whether the exponential terms in (2) really improved the prediction capability of the model.

\section{METHODS}

\section{A. Subjects}

Knee joint-lower leg dynamics was identified in three T5T6 level spinal cord injured patients (see Table I). All subjects participate in a joint research program of the Roessingh Rehabilitation Center and the University of Twente (Enschede, The Netherlands) aiming at the restoration and enhancement of paraplegic locomotion. They had normal excitable quadriceps muscle. OT's right leg quadriceps exhibited significant active spasms and was therefore not included in the experiments. The other subjects did not exhibit spasm.

\section{B Experimental Set-up}

Two experimental set-ups were used in this study. Imposed lower leg movements without quadriceps stimulation, necessary for the estimation of the inertia of the lower leg, were monitored using a dynamometer set-up. Freely swinging lower leg movements, initiated by the knee torque due to electrical stimulation of the quadriceps, were measured using a free-swing set-up. 


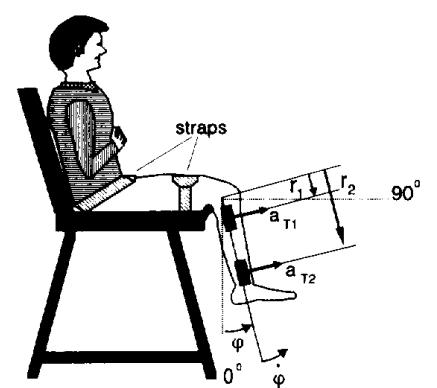

Fig. 2. Schematic of free-swing setup. The quadriceps were stimulated with pseudo-random interpulse interval sequences. The knee angle was measured with an externally mounted goniometer. Two accelerometers at radii $r_{1}$ and $r_{2}$ measured tangential accelerations (respectively $a_{T 1}$ and $a_{T 2}$ ).

Dynamometer set-up: A computer-controlled dynamometer was used for isometric recruitment curve registration and measurements to estimate the inertia of the lower leg. The subjects were seated on a KINCOM 125ES (Kinetic Communicator Exercise System) dynamometer bench (Chattecx Cooperation, Chattanooga Group, Chattanooga, TN, USA). The hip angle was fixed at approximately 100 degrees using straps. The lower leg examined was securely attached to the rotating level arm at the tibia just above the ankle. The pivot of the knee joint was aligned with the axis of the electrical motor. The knee torque was measured using a force sensor, consisting of a bridge of strain gauges on the lever arm and a built-in bridge amplifier. The knee angle and angular velocity were measured at the motor axis. The knee angular acceleration was estimated using accelerometers attached to the examined lower leg.

Free-Swing Set-up: The subjects were seated on a chair, which allowed the lower leg to swing freely (Fig. 2). The position of the subjects was identical as on the dynamometer set-up. The knee angle was measured with an externally mounted electrogoniometer. The knee angular velocity was derived off-line from the gonio signal without phase shift using digital filtering. The knee angular acceleration was estimated using accelerometers.

Angular Acceleration: Two uniaxial accelerometers (Kyowa AS-59A), fixed with soft straps on the examined lower leg in the central bone axis at two different radii $\left(r_{1}\right.$ and $\left.r_{2}\right)$ from the knee joint, measured tangential acceleration $\left(a_{T 1}\right.$ and $\left.a_{T 2}\right)$. This is depicted schematically for the freeswing set-up in Fig. 2. Knee joint acceleration $(\ddot{\varphi})$ was derived from $\left(a_{T 1}\right.$ and $\left.a_{T 2}\right)$ using the following formulas (see also [17])

$$
\begin{aligned}
a_{T 1} & =g \cdot \sin (\varphi)+\ddot{\varphi} \cdot r_{1} \\
a_{T 2} & =g \cdot \sin (\varphi)+\ddot{\varphi} \cdot r_{2} \\
\ddot{\varphi} & =\left(a_{T 1}-a_{T 2}\right) /\left(r_{1}-r_{2}\right)
\end{aligned}
$$

in which $\varphi$ is the knee angle and $g$ is the gravitational acceleration.

Stimulation and data collection: The quadriceps was stimulated using an adhesive surface cathode and anode placed over the motor point of rectus femoris and medially near the

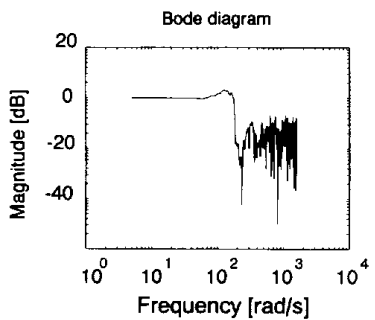

Fig. 3. Bode diagram (magnitude plot) of the force sensor on the dynamometer set-up resulted from Fourier analysis of a series of load step responses. The sensor was initially loaded with $75 \mathrm{~N}$ and decremented to $25 \mathrm{~N}$ via a step. The sensor exhibits an approximate flat response up to $15 \mathrm{~Hz}$, followed by a minor resonance peak at $25 \mathrm{~Hz}$. The half power bandwidth (i.e. the -3 $\mathrm{dB}$ point) was approximately $30 \mathrm{~Hz}$.

patella respectively. An IBM-AT compatible computer with AD facilities (Analog Devices, RTI-815, 12-bit) controlled a current stimulator, generating mono phasic rectangular pulses eliciting single muscle twitches. Pulse width (PW) remained constant at $300 \mu s$ throughout the experiment. The interpulse interval (IPI) was quantized in units of $10 \mathrm{~ms}$ corresponding to the sampling period. Recruitment was modulated by varying the pulse amplitude (PA) between 0 and $100 \mathrm{~mA} \mathrm{[18].} \mathrm{The}$ acceleration signals $\left(a_{T 1}\right.$ and $\left.a_{T 2}\right)$ were low-passed filtered with a fourth-order Butterworth anti-aliasing filter with a cutoff frequency of $35 \mathrm{~Hz}$, prior to sampling. Phase shift was compensated for off-line. The measured signals were sampled at $100 \mathrm{~Hz}$. Thus, the sampling period $T_{s}$ equaled $10 \mathrm{~ms}$. These samples and the stimulus data were stored on disk for off-line identification.

\section{Characterization of experimental set-up}

The bandwidth of the force sensor of the dynamometer setup was identified on the basis of a series of load step responses. The Fourier transform of the average of these responses was divided by the Fourier transform of a step [19]. The sensor was initially loaded with $75 \mathrm{~N}$. This load was decremented to $25 \mathrm{~N}$ via a step. The Bode diagram of the force sensor is shown in Fig. 3. The force sensor exhibited a flat response up to $15 \mathrm{~Hz}$, followed by a resonance peak at $25 \mathrm{~Hz}$. The half power bandwidth, i.e. the $-3 \mathrm{~dB}$ point, was $30 \mathrm{~Hz}$.

The accuracy and linearity of the angle and velocity sensors on the KINCOM dynamometer have been reported to be reliable [20], [21]. The external goniometer had a nonlinearity of 1 percent.

The accelerometers were examined using the dynamometer set-up. The accelerometers were rigidly attached to the lever arm at two different radii from the turning axis. Angular acceleration was derived using (3-5). Various known weights, attached to the force sensor, were moved cyclically at various speeds from -90 to $+90 \mathrm{deg}$, in counterclockwise direction according to the definition given in Fig. 1. Equation (6) describes the relation between measured signals

$$
M-G_{\text {load }} \cdot \sin (\varphi)=I_{\text {load }} \cdot \ddot{\varphi}
$$



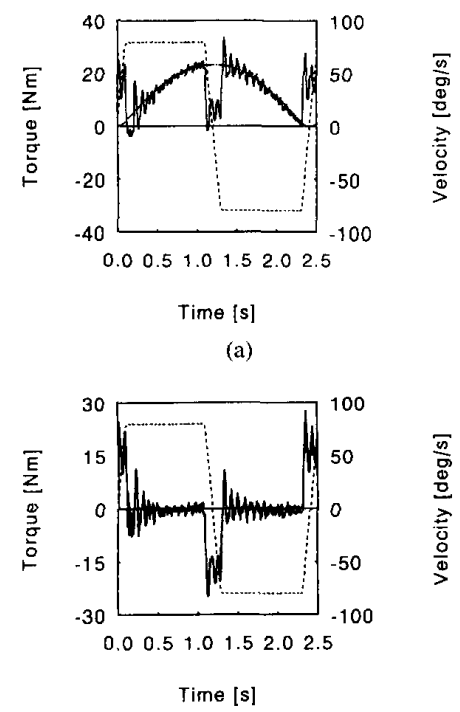

(b)

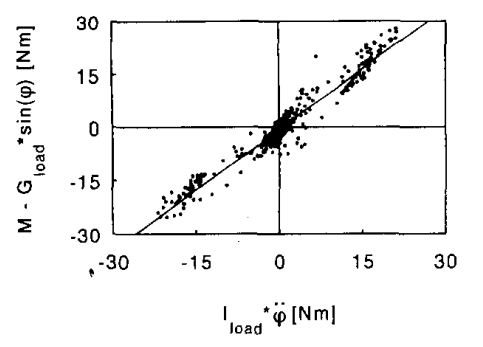

(c)

Fig. 4. (a) Measured velocity (dashed) and torque [M] (solid) during a dynamometer lever arm movement of $180 \mathrm{deg}$ with an attached load $(\mathrm{m}=5$ $\mathrm{kg}$ ). The lever arm was moved from -90 to $+90 \mathrm{deg}$, in counterclockwise direction, according to the definition given in Fig. 1. The computed torque due to gravity $\left[G_{\text {load }} \cdot \sin (\varphi)\right]$ (dash-dot) is also shown. The main deviation of the measured torque from the computed gravitational torque occurred at the turning point in the movement. At these points the electrical motor accelerated and decelerated the lever arm; (b) measured velocity (dashed), $\left[M-G_{\text {load }} \cdot \sin (\varphi)\right]$ (solid) and $[I \cdot \ddot{\varphi}]$ (dash-dot), during a lever arm movement as in Fig. 8(a). The relation between the measured signals is given in $(6)$; (c) correlation of $\left[M-G_{\text {load }} \cdot \sin (\varphi)\right]$ (y-axis) with $[I \cdot \ddot{\varphi}]$ is high $(r=0.97)$. The slope equals to 1.09

with measured motor torque $(M)$, level angle $(\varphi)$ and acceleration $(\ddot{\varphi})$, known gravity component $\left(G_{\text {load }}\right)$ and inertia of the load $\left(I_{\text {load }}\right)$. Fig. 4(a) depicts a typical registration of measured torque $(M)$ and velocity, during a cyclical dynamometer lever-arm movement with an attached mass of $5 \mathrm{~kg}$. The computed (static) torque due to the gravity $\left[G_{\text {load }} \cdot \sin (\varphi)\right]$ is also displayed. $M$ deviates from $\left[G_{\text {load }} \cdot \sin (\varphi)\right]$ at the turning points in the movement, at which the electrical motor accelerated and decelerated the lever arm. Small oscillations of the lever arm caused slight deviations during the isokinetic parts of the movement. Fig. 4(b) displays the signals [M$\left.G_{\text {load }} \cdot \sin (\varphi)\right]$ and $\left[I_{\text {load }} \cdot \ddot{\varphi}\right](6)$. The dynamic response of the accelerometers is identical to the dynamic response of the force sensor. Thus, the accelerometer set-up appeared adequate for the estimation of joint acceleration and joint torque.
Inertia: The correlation of $\left[M-G_{\text {load }} \cdot \sin (\varphi)\right]$ and $\left[I_{\text {load }}\right.$. $\ddot{\varphi}]$ were computed to investigate whether $I_{\text {load }}$ could be estimated from (6) using linear regression (Fig. 4(c)). The correlation factor $(r=0.97)$ and the linear slope (equal to 1.09 ), indicate that the inertia of a load can be estimated using combined torque and acceleration measurements and an identified parameterized relation between the measured signals.

\section{Experimental Protocol}

Successively, the following experiments were performed. The measurement systems were calibrated at the beginning of the measurement. The quadriceps muscles were warmed up by low level stimulation prior to the experiments. Two measurements were performed using the dynamometer set-up:

1. An isometric recruitment curve (knee angle of $50 \mathrm{deg}$ ) was monitored to establish the relation between PA and generated active knee torque. The IPI was $40 \mathrm{~ms}$. PA was ramped up and down from 0 to $100 \mathrm{~mA}$ and back in $20 \mathrm{sec}$ [18]. Saturation of active knee torque was obtained. PA for maximal and half recruitment (at 50\% of maximum torque) were observed.

2. For the estimation of the inertia $(I)$, various velocity steps were imposed on the knee joint-lower leg system by the electrical motor at a knee angle of 0 deg. During this measurement no stimulation was applied. Knee angular position, velocity and acceleration were recorded. Also knee torque $M_{a}$, applied externally by the electrical motor, was recorded.

Pseudo-random stimulation trials were performed on the free-swing set-up. The quadriceps of the examined leg was stimulated with three different pseudo-random IPI sequences of $5 \mathrm{~min}$ each. There was a rest period of $15 \mathrm{~min}$ in between trials. In the first two trials, PA was chosen $100 \mathrm{~mA}$, resulting in maximal recruitment. In the third trial PA was chosen to obtain half recruitment, based on the isometric recruitment curve. Additionally, several passive lower-leg swings were recorded at the end of each pseudo-random stimulation trials. Such passive swings resulted from raising the lower leg to a knee angle of approximately $50 \mathrm{deg}$ and releasing it.

\section{E. Identification Methodology and Model Prediction Evaluation}

Identification of a separate gravity component and linear stiffness (2) is very difficult because $\sin (\varphi)$ is approximately linear in the considered operating range. Also $K$ has been reported to be approximately zero in this range [14]. Therefore, $K$ was neglected. Also, in pilot sessions of the identification it was observed that the contribution of exponential term for elasticity for knee extension ( $\varphi \geq 30 \mathrm{deg}$ ) was approximately zero in the chosen operating space, by inspecting the prediction error in this region. Thus, the term with parameters $k 3$ and $k 4$ was neglected as well. Furthermore, for identification purposes, the resulting equation was divided by the inertia $I$, which was identified separately on the dynamometer set-up. 


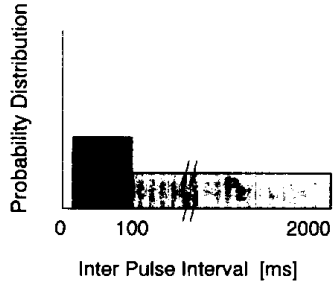

Fig. 5. Two-part uniform probability density function of the interpulse interval sequence. $P 1$ and $P 2$ are the probabilities of the two parts: $[\mathrm{P}(10<\mathrm{IPI}<100)=\mathrm{P} 1, \mathrm{P}(100<\mathrm{IPI}<2000)=\mathrm{P} 2, \mathrm{P} 1+\mathrm{P} 2=1]$. They were chosen such that the generated torque persistently excited the lower leg in the desired operating space.

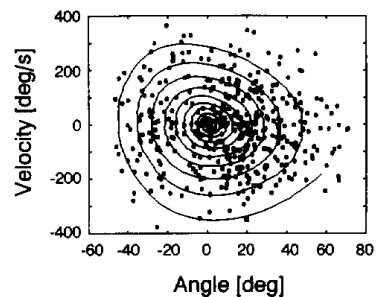

Fig. 6. Lower leg pendulum states (pairs of knee angle and angular velocity) in which the quadriceps muscle was stimulated (dots), due to the pseudo-random interpulse interval stimulation sequence. A passive swing of the lower leg is also shown (solid line) to illustrate that the lower leg system was excited in the desired knee angle range.

Thus

$$
\begin{aligned}
M_{a} / I & =\ddot{\varphi}+\left[D \cdot \dot{\varphi}+G \cdot \sin (\varphi)-k 1 \cdot\left(e^{-k 2 \cdot \varphi}-1\right)\right] / I \\
& =\ddot{\varphi}+D^{\prime} \cdot \dot{\varphi}+G^{\prime} \cdot \sin (\varphi)-k 1^{\prime} \cdot\left(e^{-k 2 \cdot \varphi}-1\right)
\end{aligned}
$$

$M_{a} / I$ will be denoted as equivalent acceleration due to quadriceps stimulation in the rest of this paper. The magnitude of $M_{a} / I$ can be estimated using (8) when the parameters $D^{\prime}, G^{\prime}$ and $k 1^{\prime}$ and $k 2$ have been identified.

Persistently Exciting Signal: A pseudo-random uncorrelated IPI stimulation signal with a two-part uniform probability density function [22] with $[\mathrm{P}(10 \leq \mathrm{IPI} \leq 100)=\mathrm{P} 1$, $\mathrm{P}(100 \leq \mathrm{IPI} \leq 2000)=\mathrm{P} 2, \mathrm{P} 1+\mathrm{P} 2=1]$ was used to generate knee torque in the free-swing set-up (see Fig. 5). Pl ensured sufficient excitation of the system in the desired knee angle operating range. Likewise, P2 made certain that passive lower leg movements were also monitored. P1 and P2 were chosen on the basis of short stimulation sessions prior to an identification trial. The system was excited by the stimulation pulses in the entire operating range (Fig. 6). Sufficient excitation is necessary to obtain a small standard deviation in the estimated parameters [19].

Identification: Passive knee joint and shank dynamics were identified using passive parts of the measured data sequence of the first pseudo-random stimulation trial on the free-swing setup. Epochs were extracted when no stimulation was applied and the muscle was completely deactivated from the previous stimulation pulse, assuming a deactivation period of $250 \mathrm{~ms}$.
Thus $M_{a} / I$ was equal to zero. The resulting equation to be identified then becomes

$$
\ddot{\varphi}=-D^{\prime} \cdot \dot{\varphi}-G^{\prime} \cdot \sin (\varphi)+k 1^{\prime} \cdot\left(e^{-k 2 \cdot \varphi}-1\right)
$$

The identification consisted of two steps repeated in an iteration loop:

Step 1. The model parameters $D^{\prime}, G^{\prime}$ and $k 1^{\prime}$ of (9) were identified using a least-squares algorithm [19], minimizing the error in estimated angular acceleration. The measured output signals of the excited lower leg system were applied as input to the identification algorithm (Fig. 7). A static nonlinearity transformed the knee angle $\varphi$ into $\sin (\varphi)$ and $(\exp (-k 2 \cdot \varphi)-1)$. The dynamic system in this cascade (9) was now linear in its inputs and has an output the estimated angular acceleration, $\ddot{\varphi}_{\text {model }}$. The total system with static nonlinearity at the input and a linear cascade dynamic system is generally referred to as a Hammerstein model [19], [23], [24] (Fig. 7). The constant parameter $k 2$ was initialized to $5.7\left[\mathrm{rad}^{-1}\right]$ based on a priori data from pilot identification sessions. In subsequent loops, $k 2$ was obtained from step 2 .

Step 2. The parameter $k 2$ was reestimated using the Levenburg-Marquardt algorithm [19]. This iteration algorithm fitted the parameters $k 2, G^{\prime}$, and $k 1^{\prime}$ of the nonlinear relation between $\ddot{\varphi}$ and $\varphi(9)$ also by minimizing the error in estimated angular acceleration. The velocity component $D^{\prime} \cdot \dot{\varphi}$ was assumed to be known from step 1. Parameters $G^{\prime}$ and $k 1^{\prime}$ needed to be estimated in this step as well to assure convergence in the estimation of $k 2$.

Thus, step 1 gave $G^{\prime}, D^{\prime}$, and $k 1^{\prime}$ and step 2 gave $k 2$. Standard deviations in the parameters followed directly from the statistical properties of the least-square estimate [19]. The loop was performed at least once. The iteration loop was stopped when convergence in the estimated parameters from step 1 was obtained. We verified the convergence of the iteration loop using computer simulation of (9) with known parameters.

The inertia of the system was estimated on the basis of the velocity step measurements on the dynamometer set-up. The measured knee angular state $(\varphi, \dot{\varphi}, \ddot{\varphi})$ and torque $M_{a}$ were substituted in the identified (8). The inertia $I$ was estimated with a linear least-squares fit.

Prediction Capability: Identification of model parameters was based on the first pseudo-random stimulation trial. The evaluation of prediction capabilities of the model were performed using the first and the two remaining pseudo-random trials. The evaluation consisted of inspecting the RMS error in the predicted angular acceleration during passive parts of the registration. Measured knee angular position $\varphi$ and velocity 


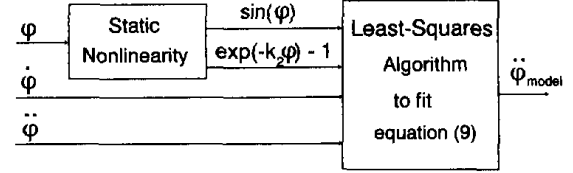

Fig. 7. Model parameters $D^{\prime}, G^{\prime}$ and $k 1^{\prime}$ were estimated using a Hammerstein model. The static nonlinearity at the input transformed the measured knee angle $\varphi$ (into $\sin (\varphi)$ and $(\exp (-k 2, \varphi)-1)$ such that the dynamic system in this cascade to be identified became linear in its inputs with output estimated joint acceleration $\ddot{\varphi}_{\text {model }}$. The model parameters were estimated using a least-squares algorithm [19], minjmizing the error in estimated angular acceleration. Parameter $k 2$ was estimated using the Levenburg-Marquardt iteration algorithm [19].

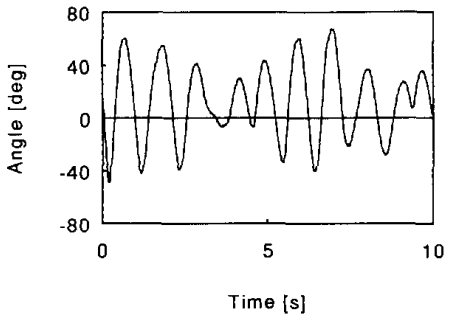

(a)

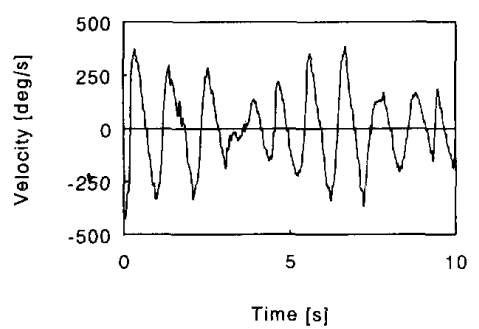

(b)

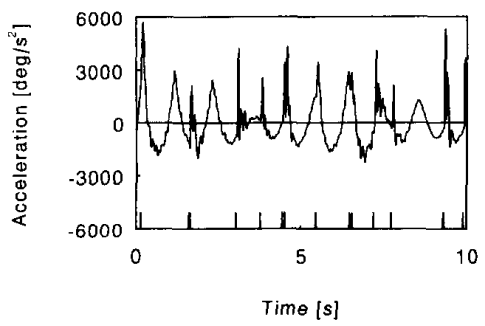

(c)

Fig. 8. Measured and estimated signals of the freely swinging lower leg system. The quadriceps were stimulated with a pseudo-random IPI stimulation sequence at maximal recruitment: (a) measured knee angle; (b) knee angular velocity estimated from the angle signal; (c) measured angular acceleration and applied stimulation pulses.

$\dot{\varphi}$ were substituted in the identified (9), which resulted in the predicted angular acceleration. The prediction capabilities of the model were also evaluated on the basis of passive swings of the lower leg. The identified model (9) was integrated forward in time for $10 \mathrm{~s}$ by steps of the sampling period, using a fourth order Runge-Kutta integration routine. The initial conditions were estimated from the first sample of the measured swing. The measured and predicted knee angle were compared quantitatively.
TABLE II

MODEL PARAMETERS OF THE KNEE JOINT - LOWER LEG SYSTEM OF THE PARTICIPATING SUBJECTS. LEFT OR RIGHT LEG IS INDICATED. THE ASTERISKS INDICATE THAT THE MEASUREMENT TO IDENTIFY THE INERTIA WAS NOT PERFORMED ON TN-RIGHT. THE SHOWN VALUES FOR THE DAMPING $D^{\prime}$, GRAVITY COMPONENT $G^{\prime}$, AND ELASTICITY PARAMETERS $k_{1}^{\prime}$ AND $k_{2}$ REFER DIRECTLY TO EQUATIONS (8) AND (9). THE APOSTROPHE INDICATES DIVISION BY THE INERTIA I. THE AVERAGE VALUES OF THE PARAMETERS WITH STANDARD DEVIATIONS (STD) ARE ALSO GIVEN. THE INERTIA WAS IDENTIFIED SEPARATELY USING THE DYNAMOMETER SET-UP.

\begin{tabular}{|c|c|c|c|c|c|}
\hline subj & $\begin{array}{c}D^{\prime} \\
{\left[\mathrm{s}^{-1}\right]}\end{array}$ & $\begin{array}{c}G^{\prime} \\
{\left[\mathrm{rad} / \mathrm{s}^{2}\right]}\end{array}$ & $\begin{array}{c}k 1^{\prime} \\
{\left[\mathrm{rad} / \mathrm{s}^{2}\right]}\end{array}$ & $\begin{array}{c}k 2 \\
{\left[\mathrm{rad}^{-1}\right]}\end{array}$ & $\begin{array}{c}I \\
{\left[\mathrm{kgm}^{2}\right]}\end{array}$ \\
\hline TN-left & 0.487 & 30.8 & 0.367 & 6.02 & 0.485 \\
\hline TN-right & 0.455 & 35.5 & 0.468 & 5.73 & $* *$ \\
\hline JM-left & 0.405 & 34.9 & 0.500 & 5.73 & 0.274 \\
\hline JM-right & 0.476 & 36.3 & 0.529 & 5.10 & 0.342 \\
\hline OT-left & 0.376 & 31.1 & 0.390 & 5.73 & 0.555 \\
\hline average & 0.440 & 33.7 & 0.451 & 5.66 & 0.414 \\
\hline std & 0.047 & 2.6 & 0.070 & 0.34 & 0.129 \\
\hline
\end{tabular}

\section{RESULTS}

\section{A. Identification of the Lower Leg Dynamics}

A typical registration of measured and estimated signals of the freely swinging lower leg system is displayed in Fig. 8. The lower leg resonated with a frequency of approximately $1 \mathrm{~Hz}$. Veltink also showed that the natural frequency of the considered system is approximately $1 \mathrm{~Hz}$ [3]. The measured angular acceleration [Fig. 8(c)] shows additional activity when stimulation pulses were applied.

Model Parameters: The model parameter values of the knee joint and shank dynamics according to ( 8 or 9$)$ of all the subjects, are given in Table II. The parameters were estimated with small standard deviations ( $\leq 2 \%$ of estimated value), indicating that the model structure components are strongly present in the observed data, its parameters are not time-dependent and are independent of each other. There is substantial intersubject variability in $I$. This intersubject variability is much less in $D^{\prime}, G^{\prime}, k 1^{\prime}$ and $k 2$ (Table II).

To test the model's sensitivity to the nonlinear joint elasticity, the left knee joint-lower leg of subject TN was also identified without the exponential term. Then $G^{\prime}$ was estimated as $36.0\left[\mathrm{rad} / \mathrm{s}^{2}\right]$ and $D^{\prime}$ as $0.99\left[\mathrm{~s}^{-1}\right]$. Thus, damping and the gravity component were estimated higher than in the model with nonlinear elasticity (compare with Table II). The standard deviations in the estimate also increased significantly.

RMS Error: The performance of the identified model (9) was quantitatively estimated by inspecting the RMS error, calculated between predicted and measured angular acceleration during measurements on the free-swing set-up (Table III). The RMS error was normalized to the RMS value of the measured acceleration. The passive parts of the first trial were used for the identification. The comparable RMS error for all trials 
TABLE III

QUANTITAVE RESLLTS OF THE PREDICTION PERFORMANCE OF THE MODEL FOR THE KNEE JOINT-LOWER LEG DYNAMICS. THE RMS ERROR BETWEEN THE KNEE ANGULAR ACCELERATION, AS MEASURED BY THE ACCELEROMETERS AND PREDICTED BY THE IDENTIFIED MODEL, WERE NORMALIZED TO THE RMS VALUE OF THE MEASURED aCCELERATION. THE RMS ERROR WAS CALCULATED USING PASSIVE PARTS OF THE REGISTRATION OF MEASURED OUTPUT SIGNALS DURING THE THREE PSEUDO-RANDOM STIMULATION TRIALS ON THE FREE-SWING SET-UP. IN THESE PARTS, NO STIMULATION WAS APPLIED AND THE MLSCLE WAS COMPLETELY DEACTIVATED FROM PREVIOUS STIMULATION PULSES. TRIAL ONE WAS USED FOR THE IDENTIFICATION,

\begin{tabular}{cccc}
\hline \multicolumn{4}{c}{ Normalized RMS error } \\
\hline subject & $\begin{array}{c}\text { (trial one) } \\
\text { maximal } \\
\text { recruitment }\end{array}$ & $\begin{array}{c}\text { (trial two) } \\
\text { maximal } \\
\text { recruitment }\end{array}$ & $\begin{array}{c}\text { (trial three) } \\
\text { half } \\
\text { recruitment }\end{array}$ \\
\hline TN-left & 0.17 & 0.16 & 0.20 \\
TN-right & 0.17 & 0.20 & 0.30 \\
JM-left & 0.17 & 0.19 & 0.14 \\
JM-right & 0.24 & 0.25 & 0.25 \\
OT-left & 0.22 & 0.20 & 0.17 \\
\hline
\end{tabular}

indicates that the identification procedure is consistent [19], [25]. The normalized RMS errors become substantially smaller $(<0.1)$ when the error signal and measured acceleration are digitally low pass filtered at $10 \mathrm{~Hz}$. This indicates that the measured acceleration could not be modeled adequately above $10 \mathrm{~Hz}$ with the proposed model structure (9). The frequency components of the knee angle and knee angular velocity above $10 \mathrm{~Hz}$ were also found to be negligible, indicating that the higher frequency components of the error were likely caused by measurement errors due to the non-rigid attachment of the accelerometers to the lower leg.

The model for TN's left lower leg dynamics, in which the nonlinear elasticity was neglected, resulted in a significantly higher normalized RMS error for the identical trials (trial one: 0.31 ; trial two: 0.30 ; trial three: 0.29 ). This indicates that the exponential term for elasticity is an essential model structure component in the considered operating space [25], and that $D^{\prime}$ and $G^{\prime}$ were wrongly estimated higher when discarding this nonlinear component.

Equivalent Acceleration: $M_{a} / I$ due to quadriceps contraction (Fig. 9) was obtained using the identified model. Each sample for $M_{a} / I$ resulted from instantaneous substitution of measured knee angle, angular velocity, and acceleration (of Fig. 8) in (8). As can be seen, activity was only detected when a stimulation pulse was applied. As discussed, the ripple on the active response (Fig. 9) was most likely caused by the non-rigid attachment of the accelerometers to the lower leg. This resulted in an overshoot in the accelerometer signals. The maximum value of $M_{a} / I$, due to a single muscle twitch, differed for the stimulation pulses (Fig. 9). This is the result of nonlinear muscle dynamics, such as torque-angle and torqueangular velocity dependencies. A typical registration of trial three, in which PA was chosen to obtain 50\% recruitment, is shown in Fig. 10. The effect of recruitment on the excitation of the system is evident when comparing Figs. 9 and 10(d). $M_{a} / I$ (Fig. 10(d)) could still be estimated by subtracting the passive contributions, identified on the basis of trial one. $M_{a} / I$ was significantly smaller, due to a stimulation pulse at $50 \%$

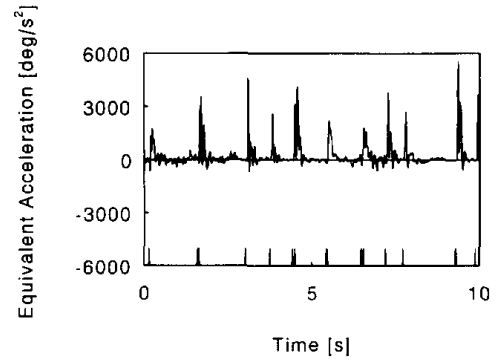

Fig. 9. Estimated equivalent acceleration $M_{a} / I$ due to elicited quadriceps contraction was obtained by subtracting the contributions of the passive system from the measured angular acceleration (Fig. 8(c)). Each sample for $M_{a} / I$ resulted from instantaneous substitution of measured knee angle (Fig. 8(a)), angular velocity (Fig. 8(b)) and acceleration (Fig. 8(c)) in the identified model (8). The stimulation pulses are also shown. The displayed sequence was cut out of the second pseudo-random trial on subject TN and was not used for the model identification. In the ideal case, $M_{a} / I$ should be zero between the stimulation pulses.

recruitment than at maximal recruitment (compare Figs. 9 and 10(d)).

Passive Swing: A typical example of the prediction of a passive swing is given in Fig. 11(a). The predicted and measured knee angle signals show high correlation. The passive swing, predicted by the model in which the nonlinear term for elasticity was neglected, is also shown. Clearly, this response extinguished more rapidly, once more indicating that the damping was estimated too high. Furthermore, it appeared that the exponential term for elasticity also affected the natural frequency of the response. The error between measured and estimated knee angle for the two models [Fig. 11(b)] emphasizes the above. Thus, including the exponential term for elasticity in the model structure, it appeared to improve the prediction in the chosen operating space.

Passive swings could be predicted accurately in all subjects using the identified model structure of (9). Typically, the important characteristics of a passive swing, such as the natural frequency and the damping [3], were estimated correctly. It was found that RMS prediction errors in the knee angle, such as depicted in Fig. 11(b) were influenced significantly by the estimated initial condition. A small error in this estimation causes a continuous phase difference between the measured and predicted passive swing, resulting in a relatively large RMS error, even though the swing characteristics were predicted accurately.

\section{DisCUSSION AND CONCLUSIONS}

The human knee joint has been modeled extensively (e.g., [26]-[31]). Most of the reported models have limited prediction capabilities, since they describe the knee joint under very restricted conditions and contain too many parameters, making them unidentifiable when only the state variables (knee angular position, velocity, and acceleration) are known. In fact, most of these models have 6 degrees of freedom while the model structure reported in the current paper is a simple 1 degree-offreedom pin joint. Thus, the reported models are not applicable for model-based joint control. In contrast, the parameterized model, as presented in this paper, is applicable for this purpose. 


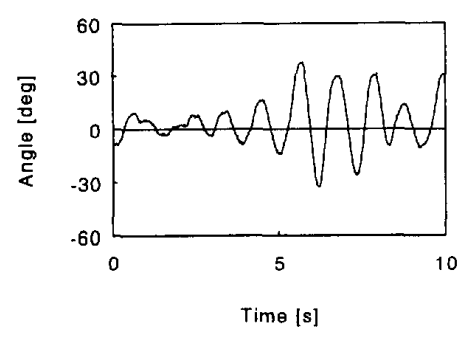

(a)

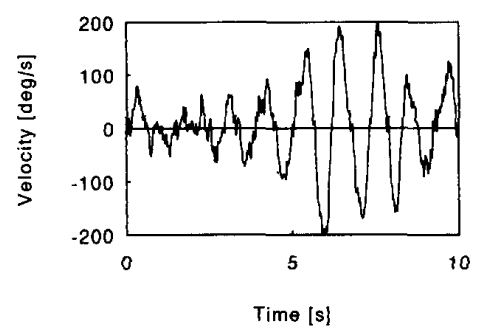

(b)

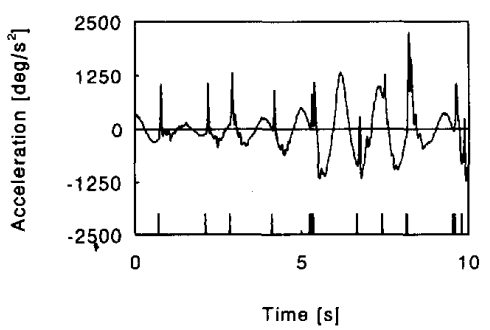

(c)

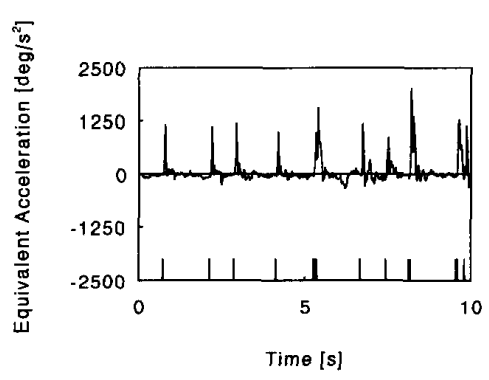

(d)

Fig. 10. Measured and estimated signals of the freely swinging lower leg system. The quadriceps were stimulated with a pseudo-random IPI stimulation sequence at $50 \%$ recruitment. The displayed sequence was cut out of the third pseudo-random trial on subject TN and was not used for the model identification: (a) Measured knee angle; (b) knee angular velocity estimated from the angle signal; (c) measured angular acceleration and applied stimulation pusles; (d) estimated equivalent acceleration $M_{a} / 1$. The methodology to obtain $M_{a} / I$ is identical as described in Fig. 9. The applied stimulation pulses are also shown.

It facilitates prediction of the total joint state. Separate joint torque contributions, due to quadriceps stimulation and knee joint-lower leg dynamics, can be accurately discriminated using the same model. Additionally, the structure contains relatively few parameters and facilitates recursive identification. The identified model simulated the knee joint acceleration with a small RMS error (normalized to the RMS value of the

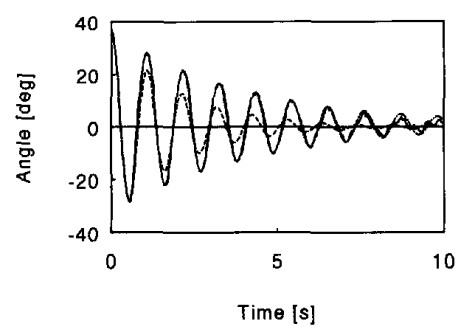

(a)

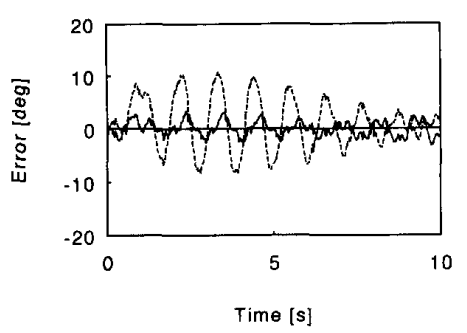

(b)

Fig. 11. (a) Example of a measured knee angle (solid) during a passive lower leg swing in patient TN-left. The predicted knee angle according to the identified passive model (9) is also displayed (dash-dot). The passive system was integrated forward in time for $10 \mathrm{~s}$ by steps of the sampling period $\left(T_{3}=10 \mathrm{~ms}\right)$ using a fourth order Runge-Kutta integration routine. The initial conditions were taken from the first sample of the measured swing. The two signals show high correlation. The predicted knee angle, according to the model in which nonlinear elasticity was neglected, is also shown (dashed). The response evidently extinguished more rapidly than the measured response. Furthermore, the phase of the response deviated from the measured response; (b) error between measured and estimated knee angle for the model according to (9) (solid) and the model in which nonlinear elasticity was neglected (dashed) in patient $\mathrm{TN}$.

measured acceleration). This RMS error became significantly smaller when low passed filtered at $10 \mathrm{~Hz}$. The accuracy in this low frequency range was confirmed by the accurate prediction of several passive swing cycles of the lower leg.

For the analysis of the movement patterns of the (paralyzed) human lower extremities several parameter values have been used for inertia, damping, gravity, stiffness and nonlinear elasticity for the passive knee joint and shank [2], [13], [15], [16]. These parameter values show large variance (e.g. [13]: $D=3.17 \mathrm{Nms} / \mathrm{rad}$; [15]: $\mathrm{D}=0.5 \mathrm{Nms} / \mathrm{rad}$ ) and were taken from references, which did not contain experimentally verified parameter values. Additionally, a component representing the gravitational torque is not mentioned in their dynamic equation [13], [15], which complicates the interpretation of remaining parameters. Published experimental data originated from measurements within a restricted operating range focused on one specific feature [14], [32]. Mansour and Audu [14] reported that the linear stiffness was very small in the operating range used in the current study. For that reason, the linear stiffness was neglected in this study. Noteworthy, paraplegic subjects with contractures might have high linear stiffness. They also pointed out that the nonlinear elasticity component near knee extension must be accounted for in the analysis of human gait. In the current study we found that the intersubject variability in $D^{\prime}, G^{\prime}, k 1^{\prime}$, and $k 2$ was small (see Table II). Additionally, it was found that the 
nonlinear term for elasticity for knee flexion contributed significantly to the lower leg dynamics in the considered operating range. If this nonlinear behavior was neglected, $D^{\prime}$ and $G^{\prime}$ were unjustly estimated significantly larger since the RMS error in predicted knee angular acceleration increased significantly. Important characteristics of a passive swing, such as the natural frequency and the damping [3], were wrongly estimated when the exponential term for elasticity was neglected. Thus, inaccurate modeling of elasticity will bias the analysis of different types of muscle models attached to the joint.

Special knee testing devices, such as reported by Robinson et al. [12] and others [2], [14], [20], [21], [32] can also be used to identify the passive knee joint and shank dynamics. These devices typically consist of a motor which drives the considered limb. Different sensor sets can be used to measure the joint angular state and joint torque. The special merit of these devices is that they allow measurements within a restricted operating range focused on one specific feature. For example, the knee joint velocity can be kept constant during cyclic movements [21]. Also, when using the device in the horizontal plane, the nonlinear gravity component can be taken out [12], [14]. Using these devices in FES applications, however, would mean more additional equipment. Driving the limb with FES-induced contraction of associated muscles and using joint angular state measurements of passive epochs to identify the passive system appeared a reasonable substitute for a special testing device. Furthermore, movements under a freely swinging condition, as investigated in this paper, cannot be studied using the reported devices.

Measurement errors contributed to the RMS error in the prediction of knee joint acceleration. The assumption that the knee joint was a perfect one-axial hinge and that the accelerometer were placed exactly in the central bone axis introduced measurement errors in knee joint acceleration (see [33] for a detailed sensitivity analysis). Additionally, in order to prevent substantial signal loss, each accelerometer was mounted on a print with a preamplifier. The weight of this combined sensor-preamplifier, had an overshoot effect on the measured acceleration due to its nonrigid attachment to the lower leg. This caused a substantial contribution to the RMS error in a frequency range above $10 \mathrm{~Hz}$.

A model for the total muscle-limb system, (i.e. the passive system consisting of passive joint and limb properties and the active system containing the muscle dynamics), could be used to predict the response of the system due to the stimulation schemes [1], [3], [6], [7], [10], [34], [35]. This is of interest for controllers trying to track the limb movement. Small disturbances can be rejected, using on-line modelbased stimulation parameter adaption. Model parameters can be identified recursively to compensate for the influence of fatigue. The identification of multiple muscle-limb systems, such as the lower extremities is, however, more complex because of additional inertial, Coriolis, and centripetal forces, arising from the dynamic interaction among the segments. It is also not sure whether the system is sufficiently excited for recursive identification during normal operation (i.e., FESinduced paraplegic gait). This limits the number of parameters which can be identified on-line. Still, the technique used in the current paper, (i.e. stimulate muscle (groups) to kinematically excite the passive system and select those parts of the measurements where no stimulation pulses were applied to identify the passive system), may also be applicable during FES-induced paraplegic gait.

\section{ACKNOWLEDGMENT}

We gratefully acknowledge the researchers of the Roessingh Rehabilitation Center, Enschede, The Netherlands. We also thank the subjects for their participation.

\section{APPENDIX}

SYMBOL TABLE

\author{
$a_{T 1}, a_{T 2}$ Tangential acceleration obtained from accelerometer 1 and 2 \\ respectively. \\ $\varphi \quad$ Knee joint angle. \\ $\dot{\varphi} \quad$ Knee joint angular velocity. \\ $\ddot{\varphi} \quad$ Knee joint angular acceleration \\ $\ddot{\varphi}_{\text {model }} \quad$ Knee joint angular acceleration estimated by the identified \\ model. \\ 9 Gravitational acceleration. \\ $k 1 . . k 4 \quad$ Parameters of the exponential function for joint elasticity. \\ $k 1^{\prime} \quad$ Parameter $k 1$ divided by the inertia of the lower leg system. \\ $r_{1}, r_{2} \quad$ Radial distance from respectively accelerometer 1 and 2 to \\ the knee joint. \\ $I$ Inertia of the lower leg system. \\ IPI Interpulse interval, i.e. the time between the onset of \\ subsequent pulses. \\ $D \quad$ Damping in the knee joint. \\ $D^{\prime} \quad$ Damping divided by the inertia. \\ $G \quad$ Gravity component of the lower leg system. \\ $G^{\prime} \quad$ Gravity component divided by the inertia. \\ $K \quad$ Linear stiffness component of the system. \\ $M_{a} \quad$ Active knee joint torque. \\ $M_{a} / I \quad$ Equivalent knee angular acceleration. \\ $P A \quad$ Amplitude of the stimulation pulses. \\ $P W \quad$ Pulse width, i.e. the duration of one stimulation pulse. \\ $T_{s} \quad$ Sampling period.
}

\section{REFERENCES}

[1] J. M. Hausdorff and W. K. Durfee, "Open-loop position control of the knee joint using electrical stimulation of the quadriceps and hamstrings," Med. \& Biol. Eng. \& Comp., vol. 29, pp. 269-280, 1991.

[2] R. E. Kearney and I. W. Hunter, "System identification of the human joint dynamics," Crit. Rev. Biom. Eng., vol. 18, no. I, pp. 55-87, 1990.

[3] P. H. Veltink, "Control of FES-induced cyclical leg movements of the lower leg," Med. \& Biol. Eng. Comp., vol. 29, pp. NS8-NS12, 1991.

[4] J. Allin and G. F. Inbar, "FNS parameter selection and upper limb characterization," IEEE Trans. Biomed. Eng., vol. BME-33, no. 9, pp. $809-817,1986$.

[5] J. Allin and G. F. Inbar, "FNS control schemes for the upper limb," IEEE Trans. Biomed. Eng., vol. BME-33, no. 9, pp. 818-828, 1986.

[6] H. J. Chizeck, R. Kobetic, E. B. Marsolais, J. J. Abbas, I. H. Donner, and E. Simon, "Control of neuromuscular stimulation systems for standing and locomotion in paraplegics," Proc. IEEE, vol. 76, 1988. pp. 1155-1165. 
[7] M. S. Hatwell, B. J. Oderkerk, C. A. Sacher, and G. F. Inbar, "The development of a model reference adaptive controller to control the knee joint of paraplegics," IEEE Trans. Biomed. Eng., vol. 36, no. 6, pp. 683-691, 1991

[8] D. R. McNeal, R. J. Nakai, P. Meadows, and W. Tu, "Open-loop control of the freely-swinging paralyzed leg," IEEE Trans. Biom. Eng., vol. BME-36, no. 9, pp. 895-905, 1989.

[9] J. J. Craig, Introduction to Robotics Mechanics \& Control, AddisonWesley Publishing Co., 1986

[10] B. Flaherty, C. J. Robinson, and G. C. Agarwal, "Determining appropriate models for joint control using surface electrical stimulation of soleus in spinal cord injury," Med. \& Biol. Eng. \& Comp., in press.

[11] B. Flaherty, C. J. Robinson, and G. C. Agarwal, "Identification of a nonlinear model of ankle dynamics during electrical stimulation of the soleus," Med. \& Biol. Eng. \& Comp., in press.

[12] C. J. Robinson, B. Flaherty, L. Fehr, G. C. Agarwal, G. F. Harris, and G. L. Gottlieb, "Biomechanical and reflex responses to joint perturbations during electrical stimulation of muscle: instrumentation and measurement techniques," Med.\& Biol. Eng. \& Comp., in press.

[13] D. T. Davy and M. L. Audu, "A dynamic optimization technique for predicting muscle forces in the swing phase of gait," J. Biomech. vol. 20 , no. 2, pp. 187-201, 1987

[14] J. M. Mansour and M. L. Audil, "The passive elastic moment at the knee and its influence on human gait," J. Biomech., vol. 19, no. 5, pp. 369-373, 1986

[15] J. M. Winters and L. Stark, "Analysis of fundamental human movement patterns through the use of in-depth antagonist muscle models," IEEE Trans. Biom. Eng., vol, 32, no. 10, pp. 826-839, 1985.

[16] G. T. Yamaguchi and F. E. Zajac, "Restoring unasisted natural gait to paraplegics via functional neuromuscular stimulation: a computer simulation study," IEEE Trans. Biom. Eng., vol. 37, no. 9, pp. 886-902, 1990.

[17] A. Th. M. Willemsen, J. A. van Alsté and H. B. K. Boom, "Real-time gait assessment utilizing a new way of accelerometry," J. Biomechanics, vol. 24, pp. 1173-1187, 1991

[18] W. K. Durfee and K. E. Maclean, "Methods for estimating isometric recruitment curves of electrically stimulated muscle, IEEE Trans. Biomed. Eng., vol. BME-36, no. 7, pp. 654-667, 1989.

[19] L. Ljung, System Identification, Theory for the User, Englewood Cliffs, NJ, Prentice Hall, 1987.

[20] M. Farrell and J. G. Richards, "Analysis of the reliability and validity of the kinetic communicator exercise machine," Med. Sci. Sport Exerc., vol. 18 , pp. $44-49,1986$

[21] H. M. Franken, P. H. Veltink, M. Fidder, and H. B. K. Boom, "Fatigue of intermittently stimulated human quadriceps during imposed cyclical lower leg movements," Int. J. of Electromyogr. and Kinesiol., vol. 3, pp. 3-12, 1993.

[22] I. W. Hunter and M. J. Korenberg, "Generation of random sequences with jointly specified probability density function and auto correlation functions," Biol. Cybern, vol. 47, pp. 141-146, 1983.

[23] S. A. Billings and S. Y. Fakhouri, "Identification of systems containing linear dynamic and static nonlinear elements, Automatica, vol. 18, no. 1, pp. 15-26, 1982.

24] I W. Hunter and M I Korenberg "The identification of nonlinear biological systems: Wiener and Hammerstein cascade models," Biol. Cybern., vol. 55, pp. 135-144, 1986

[25] K. J. Âström and B. Wittenmark, Adaptive control, Addison-Wesley Publishing Company, 1989

[26] T. P Andriacchi, R. P. Mikosz, S. J. Hampton, and J. O. Galante, "Model studies of the stiffness characteristics of the human knee joint," J. Biomech., vol. 16, no. 1, pp. 23-29, 1983.

[27] R. Crowninshield, M. H. Pope, and R. J. Johnson, "An analytical model of the knee joint," J. Biomech., vol. 9, pp. 397-405, 1976.

[28] L. Dortmans, H. Jans, A. Sauren, and A. Huson, "Nonlinear dynamic behavior of the human knee joint-part I: Postmortem frequency domain analysis.," J. Biomech. Eng., vol. 113, pp. 387-391, 1991.

[29] M. H. Moeinzadeh, A. E. Engin, and N. Akkas, "Two-dimensional dynamic modeling of the human knee joint," J. Biomech., vol. 16, no. 4, pp. 253-264, 1983

[30] G. L. Smidt, "Biomechanical analysis of knee flexion and extension," J. Biomech., vol. 6, pp. 79-92, 1973

[31] G. T. Yamaguchi and F. E. Zajac, "A planar model of the knee joint to characterize the knee extensor mechanism," J. Biomech., vol. 22, no. 1, pp. $1-10,1989$

[32] Y. F. Heerkens, R. D. Woittiez, P. A. Huijing, G. J. van Ingen Schenau, and R. H. Rozendal, "Inter-individual differences in the passive resistance of the human knee," Hum. Mov. Science, vol. 4, no. 3, pp. $167-188,1985$
[33] A. Th. M. Willemsen, C. Frigo, and H. B. K. Boom, "Lower extremity angle measurement with accelerometers-error and sensitivity analysis, IEEE Trans. Biom. Eng., vol. BME-38, no. 12, pp. 1186-1193 1991.

[34] P. E. Crago, "Muscle input-output model: the static dependence of force on length, recruitment, and firing period," IEEE Trans. Biom. Eng., vol. 39, no. 8, pp. 871-874, 1992.

[35] N. Lan, P. E. Crago, H. J. Chizek, "Feedback control methods for task regulation by electrical stimulation of muscles," IEEE Trans. Biom. Eng., vol. 38, no. 12, pp. 1213-1223, 1991.

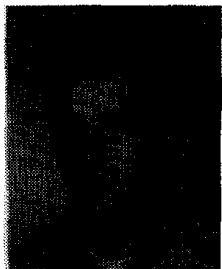

Henry M. Franken received the M.Sc. degree in Electrical Engineering from the University of Twente, Enschede, The Netherlands, in 1990 (topic: Cartesian control of a 6-link robot). He is currently pursuing the Ph.D. degree (topic: Control system design for FES-assisted paraplegic gait) at the Institute of Biomedical Technology (BMTI) of the University of Twente. His research interests involve applications of system and control engineering.

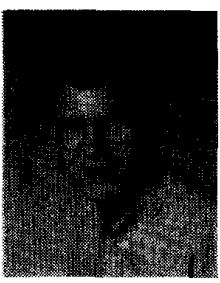

Peter H. Veltink (S'85-M'88) received the M.Sc degree in Electrical Engineering from the University of Twente, Enschede, The Netherlands, in 1984 and the Ph.D. degree in 1988 (topic: Recruitment of myelinated nerve fibers during artificial nerve stimulation).

In 1989 he was a visiting assistant professor at Case Western Reserve University, Cleveland, $\mathrm{OH}$ where he did research in the area of nonlinear control of muscle contraction. Currently, he is a faculty member of the Biomedical Engineering Departmen of the Faculty of Electrical Engineering, University of Twente. His research is in the area of control of functional neuromuscular stimulation, which is carried out in cooperation with the Roessingh Rehabilitation Center in Enschede.

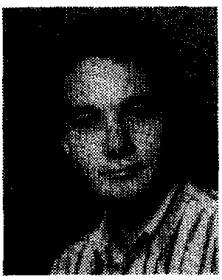

Roel Tijsmans received the M.Sc. degree in Electrical Engineering from the University of Twente, Enschede, The Netherlands, in 1993. During his study he spent three months at Strathclyde University (Glasgow, Scotland) working on FES controlled standing of paraplegics. His final project was on the modeling and identification of artificially stimulated muscle during dynamic contraction. Currently he is following a two year designer course in mechatronics.

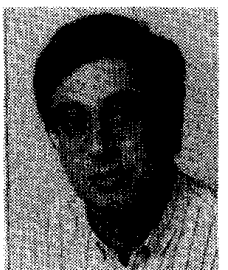

Henk Nijmeijer (M'83-SM'91) received both the university degree and doctorate in Mathematics from the University of Groningen, The Netherlands in 1979 and 1983, respectively. After spending three years at the Centre for Mathematics and Computer Science (CWI) in Amsterdam, he moved to the Department of Applied Mathematics of the Twente University of Technology in 1983, where he is presently an associate professor (UHD). His main research interests are in the area of nonlinear and linear geometric control theory and its applications. $\mathrm{He}$ is an author (with A. J. van der Schaft) of the book "Nonlinear Dynamica Control Systems," Springer (1990). Dr. Nijmeijer is a member of the editorial board of the SIAM Journal on Control and Optimisation. 


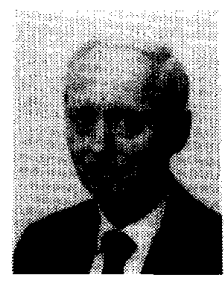

Herman B. K. Boom $\left(A^{\prime} 89\right)$ was born in 1933 and has been trained as a Medical Physicist at the University of Utrecht, The Netherlands. He received the Ph.D. ("cum laude") in 1971 and conferred on a dissertation on Ventricular Mechanics. He then joined the scientific staff of the Department of Medical Physiology of the University of Utrecht, where he was engaged in research on ventricular dynamics, and where he taught Biophysics and Physiology to medical students.

Since 1976, he has been Professor of Medical Electronics at the University of Twente, Enschede, The Netherlands, teaching Electromagnetism and Bioelectricity to Electrical Engineering students. He is directing research as chairman of the Department of Medical Information Technology of the University of Twente, which enrolls seven faculty members.

This department is part of the faculty of Electrical Engineering, and is also integrally contributing to the university's research institutes for Biomedical Technology (BMTI) and Microelectronics, Sensors, and Actuators (MESA).

$\mathrm{He}$ also chairs the Center for Rehabilitation Technology (CERT), which is a formal cooperation between the University of Twente and the Roessingh Rehabilitation Center, Enschede, The Netherlands. In this center more than 40 members of staff are participating in research on Rehabilitation Medicine and Rehabilitation Technology.

He has authored and coauthored papers on Cardiovascular Physiology, Biophysics and Technology, Bioelectricity, Muscle Dynamics, and Rehabilitation Engineering, which are also the current fields of his research interests. He has been active in the organization of international conferences, including the Annual International Conferences of the Engineering in Medicine and Biology Society. He has served as an international referee for many submitted papers and grant proposals, as well as in various committees for the evaluation of academic chairs and programs. 